This is a self-archived - parallel published version of this article in the publication archive of the University of Vaasa. It might differ from the original.

\title{
Security-constrained unit commitment with natural gas pipeline transient constraints
}

Author(s): Badakhshan, Sobhan; Ehsan, Mehdi; Shahidehpour, Mohammad; Hajibandeh, Neda; Shafie-khah, Miadreza; Catalão, João P. S.

Title: Security-constrained unit commitment with natural gas pipeline transient constraints

Year: $\quad 2019$

Version: Accepted manuscript

Copyright (C)2019 IEEE. Personal use of this material is permitted. Permission from IEEE must be obtained for all other uses, in any current or future media, including reprinting/republishing this material for advertising or promotional purposes, creating new collective works, for resale or redistribution to servers or lists, or reuse of any copyrighted component of this work in other works.

\section{Please cite the original version:}

Badakhshan, S., Ehsan, M., Shahidehpour, M., Hajibandeh, N., Shafie-khah, M., \& Catalão, J.P.S., (2020). Security-constrained unit commitment with natural gas pipeline transient constraints. IEEE Transactions on Smart Grid 11(1), 118-128. https://doi.org/10.1109/TSG.2019.2918088 


\title{
Security-Constrained Unit Commitment with Natural Gas Pipeline Transient Constraints
}

\author{
Sobhan Badakhshan, Mehdi Ehsan, Mohammad Shahidehpour, IEEE, Fellow, Neda Hajibandeh, IEEE, Student \\ Member, Miadreza Shafie-khah, IEEE, Senior Member, and João P. S. Catalão, IEEE, Senior Member
}

\begin{abstract}
The interdependencies of power systems and natural gas networks have increased due to the additional installations of more environmental-friendly and fast-ramping natural gas power plants. The natural gas transmission network constraints and the use of natural gas for other types of loads can affect the delivery of natural gas to generation units. These interdependencies will affect the power system security and economics in day-ahead and real-time operations. Hence, it is imperative to analyze the impact of natural gas network constraints on the security-constrained unit commitment (SCUC) problem. In particular, it is important to include natural gas and electricity network transients in the integrated system security because the impacts of any disturbances propagate at two distinctly different speeds in natural gas and electricity networks. Thus, analyzing the transient behavior of the natural gas network on the security of natural gas power plants would be essential as these plants are considered to be very flexible in electricity networks. This paper presents a method for solving the SCUC problem considering the transient behavior of the natural gas transmission network. The applicability of the presented method and the accuracy of the proposed solution are demonstrated for the IEEE 118-bus power system, which is linked with the natural gas transmission system and the results are discussed in the paper.
\end{abstract}

Index Terms-Natural gas transients, SCUC, gas transmission network,

\section{NOMENCLATURE}

A. Set and indexes

$i \quad$ Index of thermal generating units.

$j \quad$ Index of contingency.

$N G \quad$ Number of thermal units.

$k, y \in g \quad$ Index of node in natural gas network.

$p, q \in m \quad$ Index of bus in power transmission network.

$t \quad$ Index of hour.

$T \quad$ Number of hourly periods.

$x \quad$ Index of length through pipeline.

so Index of natural gas storage.

sup Index of natural gas supplier.

pip Index of natural gas pipeline.

B. Variables

J.P.S. Catalão acknowledges the support by FEDER funds through COMPETE 2020 and by Portuguese funds through FCT, under POCI-01-0145-FEDER-029803 (02/SAICT/2017) and POCI-01-0145-FEDER-006961 (UID/EEA/50014/2019). (Corresponding authors: Miadreza Shafie-khah and João P. S. Catalão).

S. Badakhshan and M. Ehsan are with the Center of Excellence in Management and Control of Power System, Department of Electrical Engineering, Sharif University of Technology, Tehran, Iran (e-mails: badakhshan_sobhan@alum.sharif.edu; ehsan@sharif.edu).

M. Shahidehpour is with the Robert W. Galvin Center for Electricity Innovation, Illinois Institute of Technology, Chicago, IL, 60616, USA (e-mail: ms@iit.edu).

N. Hajibandeh is with C-MAST, University of Beira Interior, Covilhã 6201001, Portugal (email: hajibandeh.n@gmail.com).

M. Shafie-khah is with the School of Technology and Innovations, University of Vaasa, 65200 Vaasa, Finland (e-mail: miadreza@gmail.com).

J.P.S. Catalão is with the Faculty of Engineering of the University of Porto and INESC TEC, Porto 4200-465, Portugal (e-mail: catalao@fe.up.pt). $\pi$

$I$

$n, f$

$P, \hat{P}$

$P_{L S}$

$Q$

$Q_{\text {com }}$

$Q^{\text {in }}, Q^{\text {out }}$

$Q_{\text {sup }}$

$\theta$

$\gamma$

C. Constants

a

$b$

$c$

$D$

DR, UR

E

$E^{\min }, E^{\max }$

$E R$

SR

$l$

MxFlow

$P_{D}$

$F^{\text {max }}$

$\pi^{\min }, \pi^{\max }$

$P^{\min }, P^{\max }$

$Q_{L}$

$Q^{\min }, Q^{\max }$

$R$

$S U, S D$

S

z

$\varphi_{f}$

$\rho$

$\alpha$

$\sigma$

$\omega$
Pressure at the nodes [bar].

Binary variable indicating ON/OFF status of units.

Startup/Shutdown binary indicator.

Generation dispatch of units [MW].

Electrical load shedding [MW].

Natural gas flow $\left[\mathrm{MSm}^{3}\right]$.

Flow of compressor [ $\left.\mathrm{MSm}^{3}\right]$.

Input/output volume of gas storage $\left[\mathrm{MSm}^{3}\right]$.

Output flow of supplier [ $\left.\mathrm{MSm}^{3}\right]$.

Voltage angle of power system buses [rad].

Voltage angle of phase shifter [rad].

Linear cost of generating units.

Constant cost of units.

Sound velocity $[\mathrm{m} / \mathrm{s}]$.

Diameter of pipeline [m].

Down and up ramp rate of units.

Storage useful inventory.

Min/Max capacity of gas storage $\left[\mathrm{MSm}^{3}\right]$.

Output rate of gas storage.

Max output of natural gas storage facilities $\left[\mathrm{MSm}^{3}\right]$.

Length of pipeline [m].

Maximum flow rate of power transmission.

Power Demand [MW].

Maximum natural gas allocation to gas fired units.

Min/max pressure of nodes in gas network [bar].

Min/max power output of generators [MW].

Gas load $\left[\mathrm{MSm}^{3}\right]$.

$\mathrm{Min} / \mathrm{max}$ output flow of supplier $\left[\mathrm{MSm}^{3}\right]$.

Spinning reserve [MW].

Fuel consumed for starting up and shutting down units.

The specific gravity of gas, it is the ratio of densities between gas and air.

Compressibility factor.

Friction factor of gas transmission.

Density of fluid (gas=0.7165).

Compression factor.

Contracted fuel price $\left[\$ / \mathrm{MSm}^{3}\right]$.

Electricity load shedding price [\$/MW].

\section{INTRODUCTION}

$\mathrm{N}$ the recent decades, the share of natural gas power plants in supplying power system loads has increased significantly. It is expected that the capacity of natural gas power plants would be increased by $110 \%$ between 2012 to 2040 [1]. 
Various factors have contributed to the fast development of natural gas power plants as compared to other electricity sources including higher efficiency, lower pollution in terms of emitting NOx, SOx and other carbon polluters, fast installation process, higher flexibility, etc. [2].

Natural gas power plants are large consumers of natural gas transmission system and large deployments of such plants can create serious issues for the natural gas network. For example, large consumptions of natural gas for supplying the static loads such as residential applications could reduce the pipeline pressure and, the reduction of natural gas supply to power plants [3].

Gas travels at a much lower velocity than electricity and due to its compressibility can be stored in pipelines. Dynamic approximations, involving gas compressibility and velocity, represent longer stabilization times required to respond to changes in gas loads and disruptions. It is undeniable that consideration of these approximations is necessary for providing the grade of flexibility and reliability that power systems require from gas. Thus, it is critical to consider natural gas network constraints and pipeline transients in power system studies.

The integrated studies of power systems which consider the effect of natural gas networks have been emphasized in the existing literature. Much of these studies have investigated the integration of natural gas systems in security constrained unit commitment (SCUC). In [4], natural gas transmission network has been implemented in the optimal power flow calculation. In general, considering natural gas transmission constraints emerges in multilateral planning of transmission systems and different types of energy hubs [5].

Various aspects of natural gas networks were introduced in power system studies. In [6], a low-carbon electricity network was investigated and in [7] energy market participation strategy was analyzed. A security constrained short-term generation planning with natural gas network was presented in [8] in which the Monte Carlo scenarios generated random contingencies on both networks. In [9], a bi-level optimization of natural gas consumption in SCUC was presented in which the natural gas network was modeled in static mode using a genetic algorithm. In [10], natural gas network was linearized and solved by the Newton-Raphson method. In [11], the variability of wind energy was firmed by the quick ramping capability of natural gas units, and Benders decomposition was adopted to check the natural gas transmission feasibility.

In [12], dynamic energy flow was optimized by different response times of natural gas and power systems. The transient response of high-pressure natural gas flow in transmission pipelines was addressed in [13] and [14], where one-dimensional flow of compressible fluid was modeled numerically. In [15], natural gas network limitations were modeled as a sub-problem in SCUC using Weymouth equations. In addition, the dynamic model of natural gas network based on determining of mass flow by modeling the gas traveling velocity, compressibility and gas pressure was implemented in SCUC [16]-[17].

The transient natural gas behavior was presented based on a set of partial differential equations (PDEs) in [18] which could pose a convergence problem in a reasonable time scale specially in the short-term power system planning where the transient behavior of the natural gas network would be more effective. In addition, exploring a reasonable solution in a ringed natural gas network could not be easily realized.

The finite difference techniques and the PDEs both in space and time can be used for the transient behavior of the natural gas in the pipe, so the behavior of the natural gas in each time step is calculated by data of the previous time step. Solving all PDEs in space and time all around the network for the transient behavior of the natural gas in the pipelines is an extremely time consuming process. The proposed model could check the feasibility of the transient behavior of the gas network by some new constraints. Such as other control systems, changing the inputs will onset the other equations.

SCUC is considered a large-scale optimization problem and introducing a set of differential equations as additional constraints to represent the transient behavior of natural gas transmission network would increase the complexity of the power system scheduling problem. In some studies, a new linear steady state model of natural gas transmission network is introduced using the Weymouth equations [19].

The increasing penetration of variable renewable energy would mandate the use of fast response and flexible natural gas plants in constrained electricity and natural gas networks [20]. The corresponding variability and the flexible operation of natural gas units, in response to variability, could initiate sudden changes in the natural gas network and affect the transient behavior of natural gas network [21].

However, the transient behavior of security-constrained natural gas network in short-term power system studies have not been widely addressed in the literature. Accordingly, we formulate the electrical analogy in this paper to consider the transient behavior of a natural gas network.

This paper seeks to model an equivalent circuit for the natural gas transmission network. The problem is solved as an electric circuit in which a power flow method is applied to an equivalent circuit of natural gas transmission pipelines. According to the transient state in the equivalent electric circuit of natural gas network, main parameters that indicate transient behavior of natural gas network could be obtained such that a certain set of constraints could be added to check the security of natural gas transmission systems in transient state. Subsequently, the natural gas network can be controlled in both transients and steady states. The modeling of the transient behavior of natural gas will be presented as a set of constraints that could be added to the Weymouth equation to check the transient stability of the gas network alongside the steady state mode of the natural gas pipeline grid.

The rest of this paper is organized as follows: Section III proposes a transient model for natural gas transmission network which is based on electrical analogy. The mathematical formulation of SCUC with natural gas transmission network is presented in Section VI. The numerical studies for the IEEE 118-bus power system with 14node natural gas transmission system is presented and analyzed in Section $\mathrm{V}$ to demonstrate merits of proposed model. Finally, the paper is concluded in Section VI.

\section{Electrical Analogy of Natural Gas Flow}

Natural gas is extracted from wells in different areas and then collected and processed via a gathering system. 
Processed natural gas is often transported through constrained pipeline networks to consumers which impose additional complexity to the natural gas flow delivery [22].

\section{A. Electrical analogy of the natural gas pipelines}

All natural gas flow equations in transient state are derived from three basic laws of mass and energy conservations and momentum [23]. These equations include mass continuity, amount of movement and energy, and an ideal natural gas status. An equivalent model of the gas networks has been presented in [24], solving the three main laws for transmission of the natural gas by replacing them with a set of equations in time and space. Therefore, they divided the pipeline to lots of small pipes and solved them along the length. In this paper, an equivalent electrical circuit has been drawn based on these three main laws and it is solved by using similar methods in the power transmission networks.

Meanwhile, the adiabatic flow assumption and constant temperature flow assumption are the most important simplifying assumptions employed for analyzing the natural gas flow inside pipelines. Analyzing the transient fluid flow of ideal compressible natural gas in a pipeline requires flow equations and implementing simplifying assumptions (such as one-dimensional isothermal flow and stable friction ratio) with reasonable errors [25]. After neglecting convective acceleration and simplifying two conservation equations (mass and energy), the following equations are obtained representing the relation of pressure and flow through horizontal pipelines. A common method is to apply the Euler finite difference technique to replace the derivative expression both in space and time. Thus, the PDEs can be transformed into a set of algebraic equations at $(\mathrm{x}, \mathrm{t})$ with time and spatial steps [26].

$$
\begin{aligned}
& \frac{\partial \pi}{\partial t}+\frac{\rho a^{2}}{A} \frac{\partial Q}{\partial x}=0 \\
& \frac{\partial Q}{\partial t}+\frac{A}{\rho} \frac{\partial \pi}{\partial x}+\frac{2 \varphi_{f} Q|Q|}{D A}=0
\end{aligned}
$$

For dynamic equations of natural gas transmission, a method has been proposed based on simulation with electricity so as to follow the well-established methods of solving electric circuits for conquering the differential equations more easily [27]. Various models describe the dynamic behavior of natural gas flow using the analogy between natural gas network and electrical network [28]. Using the analogy of voltage and current in an electrical network with pressure and flow rate in the natural gas network, lead to the electrical elements will be derived as listed in Table I [29].

Changes of flow rate depend on fluid compressibility. So, in order to consider the capacitor properties of a pipeline, the relationship between pressure, flow rate and capacity is deemed as the relationship between voltage and current within an electrical capacitor, where the current is proportionate to the incremental change of voltage.

TABLE I

ANALOGY BETWEEN NATURAL GAS AND ELECTRICAL TRANSMISSION NETWORKS

\begin{tabular}{|c|c|}
\hline Electrical & Natural Gas \\
\hline Current, I & Flow, $\mathrm{Q}$ \\
\hline Voltage, $\mathrm{V}$ & Pressure, $\pi$ \\
\hline Inductance, $L_{e}$ & Fluid Inertia Constant, $L$ \\
\hline Resistance, $R_{e}$ & Fluid resistance ratio, $R$ \\
\hline Capacitance, $C_{e}$ & Pipe capacity and compressibility, $C$ \\
\hline
\end{tabular}

The capacity effect of a pipeline network and changes in a pipeline flow rate depend on the fluid compressibility. Accordingly, the equivalent pipeline capacity is obtained as follow [30]:

$$
C=\frac{\pi r^{2} l}{\rho c^{2}}
$$

As (2) represents, pressure drop inside the pipeline might be caused by the following two factors. First, the pressure loss results from acceleration of a certain fluid mass, similar to an inductance effect in electric circuits. Second, the pressure drop might be related to frictional resistance of the pipeline. Therefore, the total pressure drop along a pipe is determined by both the resistance effect and the inductance effect. The relationship between the pressure drop and the flow change is similar to that of voltage and current at both ends of an electrical inductance [31].

Thus, the equivalent inductance of pipeline is modeled as:

$$
L=\frac{\rho l}{\pi r^{2}}
$$

Resistance estimation of a pipeline depends on the friction factor coefficient. There are several prevalent equations in the fully turbulent flow regime to estimate the Darcy friction factor, leading to distinct resistance models as presented in [32]. Moreover, the relationship between resistance and pressure drop in the pipeline conforms to Ohm's law. If the Weymouth friction factor is used, the resistance of a compressible fluid distribution system has the following form:

$$
R=R(Q, \pi)=0.6579 \times 10^{-11} \frac{T S z Q l}{D^{\frac{16}{3}}\left(\pi_{i}+\pi_{o}\right)}
$$

where the natural gas density is usually conceived as 0.7165 $\mathrm{kg} / \mathrm{m}^{3}$. In the steady state of an electric circuit, the effects of capacitors and inductors are ignored and only resistors are considered, which means capacitors and inductances are equivalent to open and closed switches, respectively. The Weymouth equation could be obtained from such an equivalent circuit of pipelines in the steady state.

Like transmission lines in a power system, an equivalent circuit is defined for each pipeline of the natural gas transmission network so that similar methods can be utilized to solve the natural gas flow [33]. One example of the equivalent electric circuit for the transmission pipeline is presented in Fig. 1. Capacitors and inductors in the equivalent circuit indicate different time constants in the dynamic behavior of the fluid flow in pipelines due to the storage and compressibility of natural gas. The modeling of this equivalent circuit is obtained based on the steady state behavior of natural gas transmission network.

\section{B. Transient behavior of natural gas network}

Natural gas is transmitted from suppliers to consumers through transmission pipelines in a similar way to the delivery of power from power plants to consumers through transmission lines. However, different nature of natural gas and electricity leads to various differences in their transmission characteristics. Natural gas even has many transmission disparities with other fluids due to its compactness [34].

After the electricity transmission network, natural gas transmission networks are the second largest energy transmission networks in size. 
However, due to compressibility properties of natural gas, natural gas networks are considered as the most complex nonlinear systems in operation [35]. Also, natural gas power plants are the coupling points of these two largest energy transmission networks. Moreover, the operating condition of each network affects the other so that it is necessary to study the two networks in an integrated manner. Given the equivalent circuit for transmission pipelines, the two networks can be modeled in a unified form but solved differently. As shown in Fig. 1, in this analogy between natural gas transmission pipes and electricity elements, adding a natural gas power plant to one end of a pipeline is equivalent to enforcing a step input signal in the equivalent electrical circuit.

One of the assumptions about using the analogy between the power transmission network and the natural gas pipeline is that each change in the pressure and flow of the pipelines is like a step-one function and other variable parameters will be calculated based on it. Indeed, the proposed model has no independency to time duration. If the flow at the end of natural gas transmission network is known as natural gas load and the pressure at the beginning is set as a reference, as shown in Fig. 2, the equivalent circuit could be modeled as two equations with two unknowns.

The mathematical equation for equivalent electrical analogy of natural gas pipelines is stated in the following form with pipeline pressure as output:

$$
\left[\begin{array}{l}
\pi_{i} \\
Q_{i}
\end{array}\right]=\left[\begin{array}{cc}
1+\frac{Z Y}{2} & Z \\
Y\left(1+\frac{Z Y}{4}\right) & 1+\frac{Z Y}{2}
\end{array}\right]\left[\begin{array}{l}
\pi_{o} \\
Q_{o}
\end{array}\right]
$$

Accordingly, a large-scale second-order circuit with various inputs would be created for a natural gas transmission network. However, it is very time-consuming and difficult to solve the pertinent set of second-order differential equations, especially for large-scale natural gas networks.

To find the transients of natural gas transmission based on the proposed electrical analogy, we solve the circuit by using the Laplace transform and specifying specific properties of inputs. After the Laplace transform, the following parametric equations are obtained:

$$
\begin{aligned}
& Z=R(Q, \pi)+S L \quad Y=S C \\
& \pi_{1}=\frac{\pi_{S}}{S} \quad Q_{2}=\frac{Q_{S}}{S}
\end{aligned}
$$

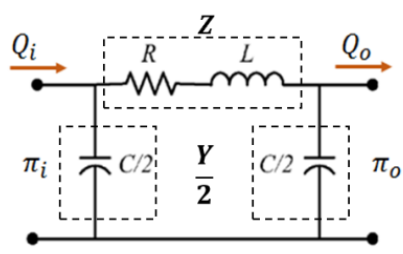

Fig. 1. Pi-Equivalent Circuit for dynamic fluid movement in transmission pipelines.

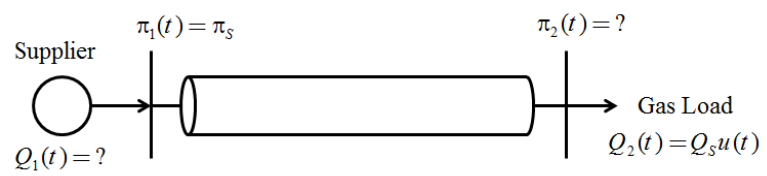

Fig. 2. Operation inputs of a pipeline in a natural gas network.

$$
\begin{aligned}
\pi_{2} & =\frac{2 \pi_{S}-2(R+S L) Q_{S}}{S\left(L C S^{2}+R C S+2\right)} \\
Q_{1} & =\frac{4 Y P_{1}(t)+Z Y^{2} P_{1}(t)+4 Q_{2}(t)}{4+2 Z Y}
\end{aligned}
$$

The following variables are defined for simplifying these equations:

$$
\begin{aligned}
\lambda & =P_{S}-R Q_{S} \\
\beta & =\sqrt{\frac{2}{L C}-\frac{R^{2}}{4 L^{2}}} \\
\gamma & =\frac{R}{2 L}+\frac{2 Q_{S}}{\lambda C} \\
\mu & =\frac{R C}{L} \pi_{S}-2 Q_{S} \\
\chi & =\frac{4 \pi_{S}-2 Q_{S} R}{L} \\
\varphi & =\frac{D}{C \pi_{S}}-\frac{R}{2 L}
\end{aligned}
$$

Finally, after transforming the Laplace equations in the frequency domain back to the time domain, we can obtain the transients of natural gas flow at the beginning of a pipe and pressure at its end.

$$
\begin{aligned}
\pi_{2}(t)=\lambda(u(t) & \left.-e^{-\frac{R}{2 L}} \cos (\beta t)-\frac{\gamma}{\beta} \sin (\beta t)\right) \\
Q_{1}(t)=Q_{S} u(t)+ & \frac{\chi}{2 \beta} e^{-\frac{R}{2 L} t} \sin (\beta t) \\
& +\frac{C P_{S}}{2}\left(e^{-\frac{R}{2 L}} \cos (\beta t)\right. \\
& \left.-\frac{R}{2 L \beta} e^{-\frac{R}{2 L} t} \sin (\beta t)\right) \\
& \times\left(e^{-\frac{R}{2 L} t} \cos (\beta t)\right. \\
& \left.+\frac{\varphi}{\beta} e^{-\frac{R}{2 L} t} \sin (\beta t)\right)
\end{aligned}
$$

The start of a gas-fired unit in one side of a pipe could be modeled as a step-one input to the equivalent electrical circuit of the pipeline. According to this analogy, the transient behavior of the other variable is obtained in Eq. (17)-(18).

Based on these two equations, the dynamic behavior of natural gas transmission can be analyzed and observed parametrically. Sudden changes of the operating state in a pipe spread across the transmission network as a moment with the acoustic speed until the stabilization of all the pressure in the network. The exponential terms in the equations show the damping and response delay of the natural gas network.

\section{EXTENDED SECURITY CONSTRAINED UNIT COMMITMENT}

\section{A. Security-constrained unit commitment}

Security-constrained unit commitment (SCUC) is widely used for minimizing power system operating costs while ensuring the security of the determined scheduling [36]. The SCUC is commonly formulated as a mixed integer programming problem subject to prevailing constraints in power system operations.

The objective function that minimize the cost of scheduling thermal generating units is presented below: 


$$
\begin{gathered}
\operatorname{Min} \sum_{t=1}^{T} \sum_{i=1}^{N G} \sigma_{i}\left(a_{i} P_{i, t}^{2}+b_{i} P_{i, t}+c_{i} I_{i, t}+S U_{i, t}\right. \\
\left.+S D_{i, t}\right)+\sum_{t=1}^{T} \omega P_{L S}
\end{gathered}
$$

Common operational and technical constraints include system energy balance, ramp up/down rate limits, system spinning/operating reserve requirements, system emission limits, unit output limits, min On/Off time limits, transmission line flow limit, emission limits, and fuel limits. Detailed formulation of SCUC and the related constraints are described in [37]. The nonlinear generation cost is linearized in the following equations:

$$
\begin{aligned}
& P_{i}=\sum_{l=1}^{L} \Delta P_{i}(l) \quad \forall i \\
& o \leq \Delta P_{i}(l) \leq \frac{P_{i}^{\text {max }}}{L} \quad \forall i, l=1,2, \ldots, L \\
& C G_{i}=\sum_{l=1}^{L} k_{i}(l) \Delta P_{i}(l)+c \quad \forall i \\
& k_{i}(l)=(2 l-1) a_{i}\left(\frac{P_{i}^{\text {max }}}{L}\right)+b_{i} \quad \forall i, l=1,2, \ldots, l
\end{aligned}
$$

Spinning reserve limits:

$$
\sum_{i=1}^{N} P_{i}^{\max } I_{i, t} \geq P_{D, t}+R_{t}
$$

Ramping rate limits:

$P_{i, t}-P_{i, t+1} \leq D R_{i}\left(1-n_{i, t}\right)+n_{i, t} P_{i}^{\min } \quad \forall i, \forall t$

$P_{i, t+1}-P_{i, t} \leq U R_{i}\left(1-f_{i, t}\right)+f_{i, t} P_{i}^{\text {min }} \quad \forall i, \forall t$

Max/min generation output:

$P_{i}^{\text {min }} I_{i, t} \leq P_{i, t} \leq P_{i}^{\text {max }} I_{i, t}$

Minimum ON time constraints:

$f_{i, t} \leq I_{i, t+T U(i, e)} \quad \forall i, \forall t$

Where, TU $(\mathrm{i}, \mathrm{e})$ is presented as follows:

$T U(i, e)=\left\{\begin{array}{ll}e & e \leq T_{i}^{o n} \\ 0 & e>T_{i}^{o n}\end{array} \quad \forall i\right.$

Minimum OFF-time constraints:

$n_{i, t}+I_{i, t+T D(i, e)} \leq 1 \quad \forall i, \forall t$

Where TD (i,e) is presented as follows

$T D(i, e)=\left\{\begin{array}{ll}e & e \leq T_{i}^{\text {off }} \\ 0 & e>T_{i}^{\text {off }}\end{array} \quad \forall i\right.$

Natural Gas consumption constraint:

$a_{i} P_{i, t}^{2}+b_{i} P_{i, t}+c_{i} I_{i, t} \leq F_{i}^{\max } \quad \forall i, \forall t$

As objective function, this equation will be linearized according to (20)-(23).

Power transmission constraint in normal conditions:

$$
\begin{aligned}
& a_{p q}\left(\theta_{p, t}-\theta_{q, t}-\gamma_{p q, t}\right) \\
& \leq M x \text { Flow }_{m} \quad \forall m,(p, q \in m), t
\end{aligned}
$$

Power transmission constraint in contingency conditions:

$$
\begin{aligned}
a_{p q}^{(j)}\left(\theta_{p, t}-\theta_{q, t}-\right. & \left.\gamma_{p q, t}\right) \\
& \leq M x F \operatorname{Mlow}_{m}^{(j)} \quad \forall m,(p, q \\
& \in m), j, t
\end{aligned}
$$

Contingencies are related to some lines and elements that have a dominant effect on the operation and topology of the power system and they could be single or multi-contingency based on the importance of the element and according to the related experience about the operation of each power network.

B. Natural gas storage

There are two ways for compressible natural gas to be stored in transmission networks for future use during critical periods [38]: directly stored in pipelines or kept in alternative storage tanks. A model has been designed for optimizing the remaining natural gas volume in the transmission pipes, which in fact determines the difference between the input and output natural gas at the beginning and at the end of each natural gas pipe [39]. For achieving a higher modeling accuracy and coordinating the two natural gas storage options, we resort to using dynamic and transient-based modeling methods.

In order to maintain the security and smoothness of the steady state natural gas flow, large quantities of natural gas are stored in storage tanks. Only when a contingency occurs on a natural gas transmission pipeline in a peak period, those storage facilities are committed and added to the natural gas transmission network. Accordingly, natural gas storage is modeled as a temporary supplier of natural gas, whose output rate and storage volume are given as follows:

$$
\begin{aligned}
& E_{i, t+1}=E_{i, t}+Q_{s o, t}^{\text {in }}-Q_{s o, t}^{\text {out }} \times E R_{s o} \quad \forall s o, \forall t \\
& E^{\text {min }} \leq E_{t} \leq E^{\text {max }} \quad \forall t
\end{aligned}
$$

where the output rate limit and tank capacity are available from natural gas companies.

\section{Compressor}

A compressor is analogous to a transformer that changes the voltage level in power systems. A simplified modeling of compressors is available in [22], which determines the quantity of natural gas compressed at each node according to a specified compressor factor. So, the transmission matrix modeling the relation of output and input parameters of a compressor is presented in the following:

$$
\left[\begin{array}{l}
\pi_{i} \\
Q_{i}
\end{array}\right]=\left[\begin{array}{ll}
\alpha & 0 \\
0 & 1
\end{array}\right]\left[\begin{array}{l}
\pi_{o} \\
Q_{o}
\end{array}\right]
$$

For pipelines with compressors, the pressure at the incoming end can be lower than that at the outgoing end.

\section{Natural gas transient modeling}

Natural gas fuel limits are modelled in natural gas load flow constraints. The natural gas load flow equation based on electrical analogy is formulated as follows [19]:

$$
\begin{gathered}
q_{k y, t}=\operatorname{sign}\left(\pi_{k, t}, \pi_{y, t}\right) \sqrt{\left(\pi_{k, t}^{2}-\pi_{y, t}^{2}\right)} \quad \forall g,(k, y \\
\in g), t
\end{gathered}
$$

As shown in Fig. 4, the total sum of all inputs and outputs at each natural gas node should be zero. Thus, the balance constraint of fluid flowing through pipelines at each natural gas node is represented in the following:

$$
\begin{aligned}
\sum_{\text {sup }=1}^{S U P} Q_{\text {sup }}+\sum_{\text {so }=1}^{S T O} Q_{s o}-\sum_{d=1}^{D E M} G L_{d} & \\
& =\sum_{L=1}^{P I P} Q_{L}+\sum_{\text {com }=1}^{C P R} Q_{c o m} \quad \forall t
\end{aligned}
$$


Other operation constraints of natural gas network include pressure limits of each node and output limit of natural gas suppliers as written below:

$$
\begin{array}{ll}
\pi_{g}^{\text {min }} \leq \pi_{g, t} \leq \pi_{g}^{\text {max }} & \forall g, \forall t \\
Q_{\text {sup }}^{\text {min }} \leq Q_{\text {sup }, t} \leq Q_{\text {sup }}^{\text {max }} & \forall \text { sup }, \forall t \\
Q_{\text {so }, t}^{\text {in }}, Q_{\text {so }, t}^{\text {out }} \leq S R_{\text {so }} & \forall s o, \forall t
\end{array}
$$

With the natural gas storage equations obtained in section III-B, transient overshoot of the network and damping time of each pipe can be calculated for evaluating the transient stability based on steady state values.

An identical behavior of the natural gas in the pipeline is shown in Fig. 3, where the outputs follow any change in the network without any unstable behavior and transient pressure drop. From the SCUC operator point of view, the definition of the decision variable to present a reliable scheduling in both the power system and the natural gas networks is more important than seeing the behavior of the planning during time. So these decision variables for controlling the transient behavior are formulated in Eq. (40)-(43)-(45), being able to respond to any change in the condition of the network.

Natural gas networks never exhibit fluctuating transient behavior due to their physical structure and operation timescale; in most cases, instantaneous load changes result in pressure drop. Therefore, the frequency of transient response for modeling the dynamic behavior is considered zero.

Accordingly, transient state equations based on electrical analogy are simplified as follows:

$$
\begin{aligned}
& \beta=0, \quad \frac{8 L}{C}=R^{2}(\pi, Q) \\
& \pi_{2}(t)=\lambda\left(u(t)-e^{-\frac{R}{2 L} t}-\gamma t e^{-\frac{R}{2 L} t}\right)
\end{aligned}
$$

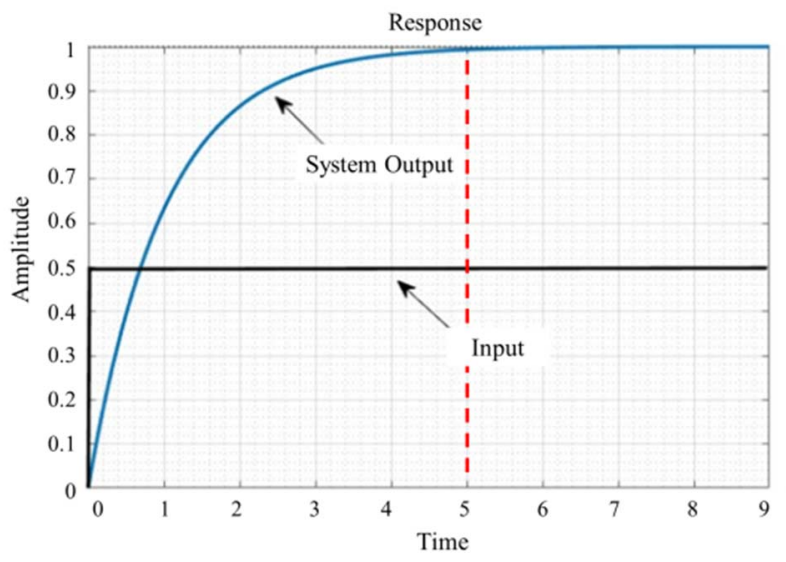

Fig. 3. Identical transient behavior of the gas network output.

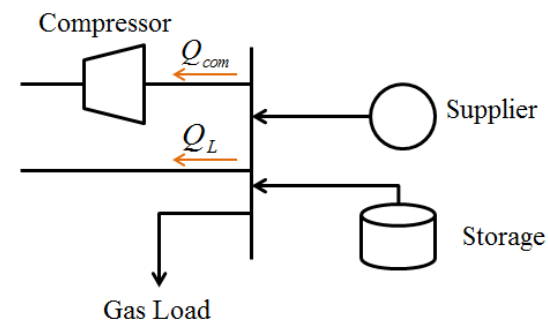

Fig. 4. Different flows in natural gas node.

$$
\begin{aligned}
Q_{1}(t)=Q_{S} u(t)+ & \frac{\chi}{2} t e^{-\frac{R}{2 L} t}+\frac{C P_{S}}{2}\left(e^{-\frac{R}{2 L}}\right. \\
& \left.-\frac{R}{2 L} t e^{-\frac{R}{2 L} t}\right) \times\left(e^{-\frac{R}{2 L}}+\varphi t e^{-\frac{R}{2 L} t}\right)
\end{aligned}
$$

which do not exhibit instantaneous pressure increase with undershoot considered in accordance with the real-life natural gas network operation. Furthermore, the existence of the exponential term and their time constants could show the amount of natural gas that has been stored in the pipelines, known as line pack effect.

On the other hand, SCUC is implemented hourly and each time period of scheduling is 1 hour. Steady state equations of a natural gas network are only valid when the network's transient behavior is less than 1 hour so that we create another constraint on scheduling validity by controlling the time constant of transient state equations less than 1 hour (shown in Fig. 3, with red dashed line).

$$
\frac{3}{\tau}=\frac{6 L_{p i p, t}}{R_{p i p, t}(\pi, q)} \leq 3600 \quad \forall t, \forall p i p
$$

Maximum pressure drop and transient flow of the natural gas network can be calculated in order to preserve the network security during transients. Specifically, the maximum pressure drop should be limited such that natural gas load variations do not cause significant transient nor instantaneous pressure drop for making the network operation unstable. The maximum transient pressure drop is represented as follows:

$$
\begin{aligned}
& T\left(\pi_{\text {min }}\right)=\frac{1}{\gamma}-\frac{2 L_{\text {pip }, t}}{R_{\text {pip }, t}(\pi, q)} \quad \forall t, \forall p i p \\
& \pi_{2}\left(T_{\text {min }}\right) \geq \frac{1}{2} \pi_{\text {min }}
\end{aligned}
$$

To this end, constraints (40), (43), and (45) are added to the SCUC model to ensure transient security of the natural gas network pertinent to the obtained generation schedule.

E. Complete solution process

Cooperation between the ISO or the utility operator and natural gas transmission operators is based on the exchange of the information of industrial and residential gas load forecasting with service priorities, natural gas transmission parameters, and planned outage of natural gas pipelines with ISO that would execute the day-ahead SCUC. It will check the feasibility of the natural gas transmission system for serving the expected natural gas loads based on available natural gas resources.

Transient behavior of natural gas network is investigated based on steady state electrical analogy such as the transient resistance. In other words, after determining steady state behavior of the natural gas network, equivalent resistance is calculated by using steady state pressure and flow as well as parameters for determining transient behavior. In other words, allowed values for sudden overshoot or undershoot in the natural gas network and allowed time to stabilize the natural gas network after disturbance can also be added for controlling transient security of natural gas network.

The SCUC problem is MIP and the natural gas transmission system in the steady state and transient stability check is nonlinear. 
The solution method includes two levels. The first level uses a GA algorithm to solve the natural gas network problem and the second level uses the CPLEX solver in order to solve the SCUC optimization problem. The first level is a heuristic method to solve the nonlinear problem of the gas network system. A mixed integer linear programming problem is associated with the second level of the optimization problem (SCUC problem). In the first level the natural gas allocated to gas-fired units are variables of the problem. The GA will provide an initial value for these variables that could cover all natural gas transmission network constraints based on other natural gas network input data, such as other gas loads. The allocated gas will be sent to the SCUC problem. The operation cost of the power network will be the first value of the objective function of the GA, and in the other levels the problem will change the natural gas allocation to gas-fired units to find the minimum operation cost. This process will be checked in some different hours of the gas network with different conditions to find the best solution. In this way, all of the chromosomes which are sent to the second level problem are feasible in the viewpoint of the gas network.

These two problems have been separated as mentioned in [9], while a genetic algorithm is used for the nonlinear equation of the natural gas system. Thus, generation schedules obtained from steady state Weymouth equations can be investigated under transient security constraints of the natural gas network to ensure their dynamic stability.

\section{CASE STUDY}

Performance of the proposed SCUC model has been tested on the IEEE 24-bus power system and Belgian 20-node natural gas transmission system. The topology of the integrated power and natural gas network is shown in Fig. 4.

The power system has 24 buses, 38 branches, 17 loads, and three natural gas-fired units; four oil-fired units, five photovoltaic units; and three hydro units. The natural gas network has 20 nodes, 21 pipelines, 2 suppliers, 4 storages, and 9 non-electrical loads. All domestic demands and commercial natural gas loads are prior to electrical gas loads and it is assumed that they have firm transport contracts. Natural gas power plants have interruptible transport contracts. Complete configuration details about the test network are available at [16].

In order to evaluate the efficiency of the proposed model with transient constraints of natural gas transmission, we consider three distinct cases and compare the resulting generation schedules. In the first case, SCUC is solved only using steady state constraints of natural gas network. In the second scenario, SCUC is solved using transient state constraints in addition to steady state constraints. Since the operation of natural gas networks is affected by uncertainties of renewable energy generation and sudden changes of natural gas flow; thus, in the last case, the penetration rate of renewable energy resources is increased to investigate the effect of transient equations, when SCUC is solved and analyzed during transients and steady states separately. All domestic demands and commercial natural gas loads are assumed with a higher priority to electrical natural gas loads. All of them which have firm transport contracts and the supply to natural gas power plants is interruptible.

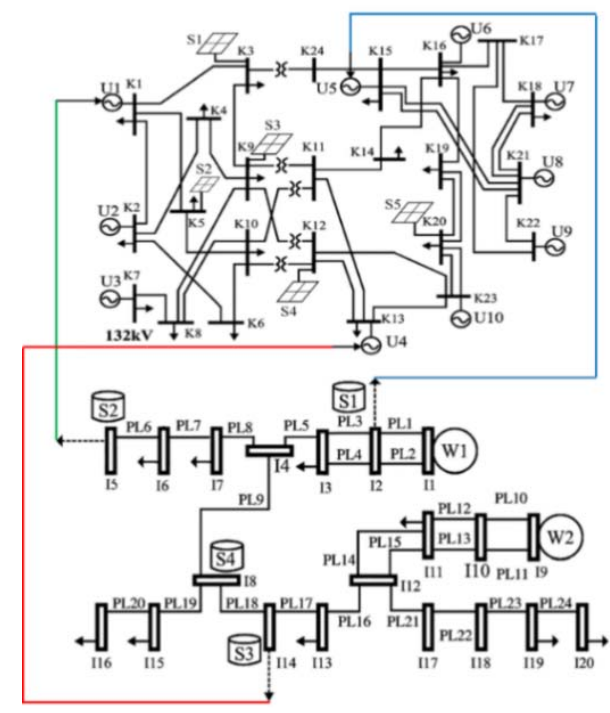

Fig. 4. Topology of 24-bus power system linked with natural gas transmission network.

Case 1: SCUC with Steady State Constraint of natural gas transmission

Daily operation cost of the power system with natural gas transmission constraints in steady state is $6.135841 \mathrm{M} \$$. For this purpose, the generation size and population of GA are assigned to 100 and 250 , respectively. The stall generation of 180 is used as stopping criterion, setting the objective function tolerance to $1 \$$. The Gaussian is used as mutation function. The proposed model used a $2.63 \mathrm{GHz}$ Intel processor with 4 GB of memory. The Gaussian is used as mutation function.

Case 2: SCUC with Steady State and Transient Constraint of natural gas transmission

The power system operation cost is $6.269804 \mathrm{M} \$$ after solving the proposed model for natural gas load flow.

After linearizing natural gas flow equations, obtained values (i.e., pressure, flow) are added to transient state constraints. If the transient constraints are met, final solution is obtained; otherwise, the SCUC model is solved again and generation schedules would be changed to obtain final solution of the problem.

Comparison of the generation schedules obtained from the first two cases is shown in Fig. 5.

As can be seen in Fig. 5, the introduction of transient state constraints reduces sudden changes to the least and natural gas network stability becomes less sensitive to load changes. Considering the inertia of natural gas in transmission network, this stable behavior contributes to the operational performance of the natural gas network in practice.

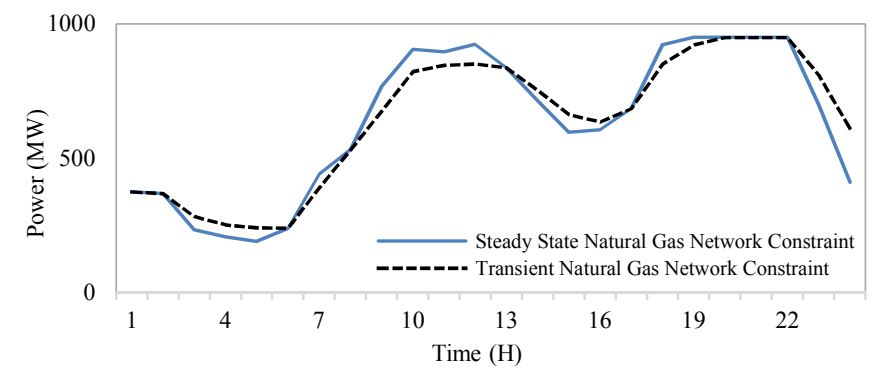

Fig. 5. Natural Gas consumption in power plants with steady state and transient gas network constraints. 
In Fig. 6, the generation schedule of Unit 5 is presented. As shown, transient constraints of natural gas network cause the power plant to use more natural gas from the natural gas stored in pipelines at some hours, but the power plant output is reduced at some hours because of the new transient state constraints. Comparison of total natural gas consumption in the power system with and without considering transient gas constraints is shown in Fig. 7. Natural gas consumption of power plants increases when transient behavior of natural gas network is considered, while the natural gas withdrawal from suppliers is approximately equal in these two cases. That is because the transient study models the natural gas stored in pipelines. Considering transient state constraints, the total amount of daily natural gas consumption in natural gas power plants reduces the sudden changes of hourly natural gas loads of these power plants (about a few percent's in this case), which represents the volume of natural gas that would be stored in the natural gas pipelines.

Case 3: SCUC with Steady State and Transient Constraint of natural gas transmission in large scale system

Now the proposed method will be tested on a large-scale power system network linked to a natural gas transmission grid. A modified IEEE 118-bus system linked with 14 node natural gas transmission grid as shown in Fig. 8, and Fig. 9, respectively, is studied. The power system has 12 combined cycle units, 54 fossil units, 7 hydro units, 9 tap changing transformers, 186 branches, and 91 demand sides. The natural gas transmission has 12 pipelines, 14 nodes, and two compressors. The complete test data is given in [40]. It is assumed that generation of $1 \mathrm{MBtu}$ of electrical energy consumes 1 kilo cubic feet of natural gas. At first, SCUC is calculated with natural gas constraints based on Weymouth equations in steady state, then the model is extended with transient constraints of natural gas network. The results are compared in Fig. 10.

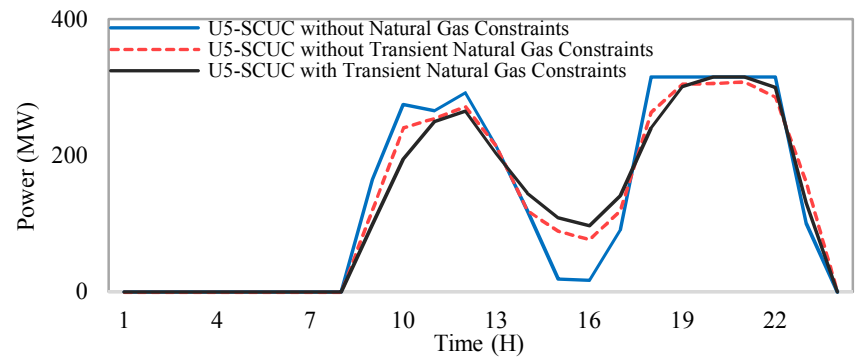

Fig. 6. Scheduling of U5 in normal mode and with Natural gas Transmission Network.

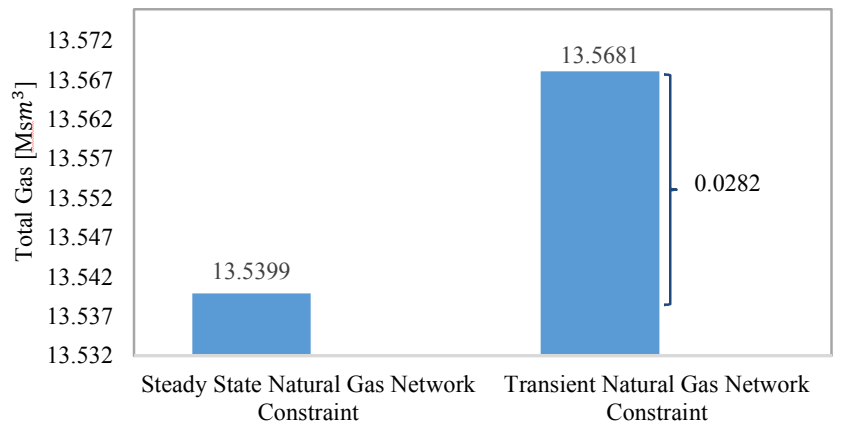

Fig. 7. Natural Gas Consumption with static and transient Natural Gas Constraints.

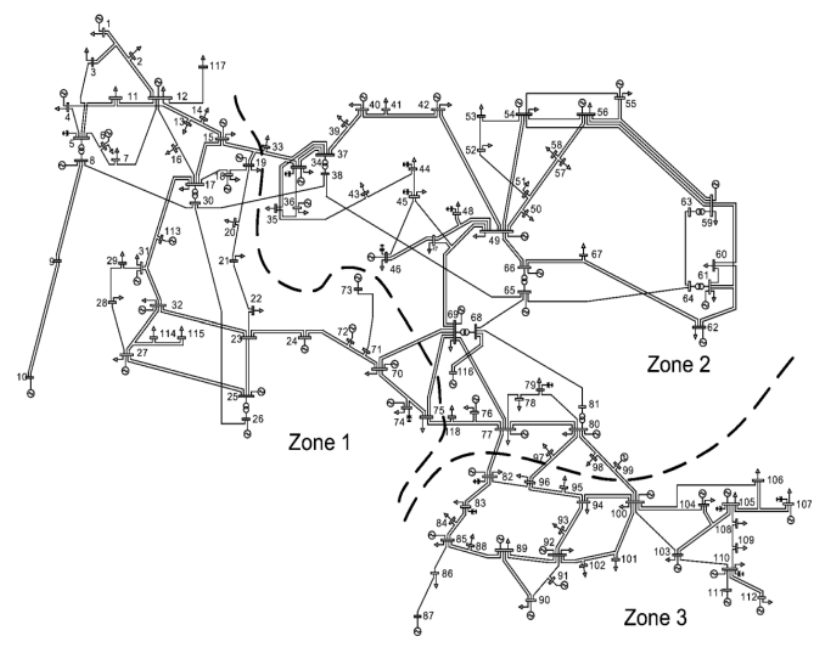

Fig. 8. 118-bus power transmission system.

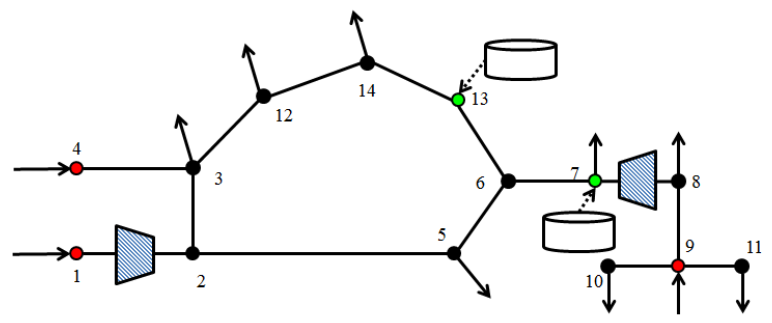

Fig. 9. Topology of natural gas transmission system.

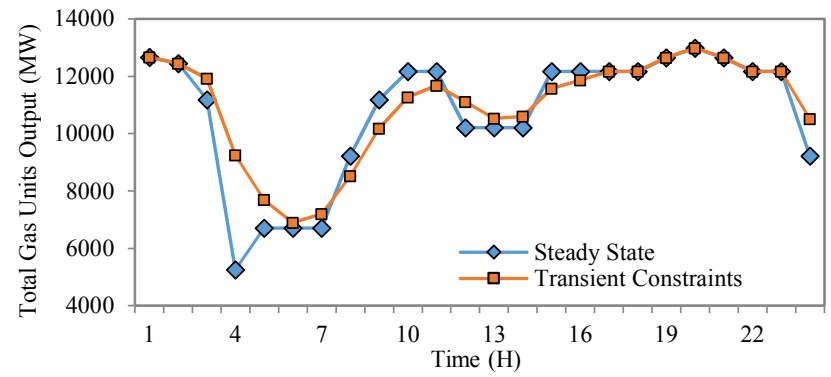

Fig. 10. Natural gas-fired units output with steady state and transient gas network constraints.

Transient constraints of the natural gas grid inhibit from the sudden decrease and increase in the gas allocation to power plants, and it is shown in some cases to be unsatisfying in the transient constraints that could change the steady state results. In other words, the transient stability feasibility check of the gas grid needs steady state results that were based on the Weymouth recalculated and finally reschedule the output of different power plants in SCUC.

\section{CONCLUSION}

A SCUC model with transient gas transmission network constraints was proposed in this paper to utilize the electrical analogy between power transmission line and natural gas flow in the pipeline. Accordingly, a new set of constraints are defined for the transient stability of natural gas network. In order to check the transient stability of the natural gas grid, steady state parameters of the natural gas network, such as pressure and flow of pipelines, are embedded in the proposed transient state constraints. Unlike other methods, our method avoids complex and time-consuming calculations in 
large-scale systems for natural gas flow, especially in shortterm operation planning of power systems, such as the dayahead scheduling of thermal power plants. Case studies have demonstrated the effectiveness of the proposed model based on the IEEE test power system linked by the natural gas grid. Although this is an appropriate method for the large-scale natural gas transmission network, one of its most important advantages is that this method can be easily interfaced and linked with other prevalent optimization problems for power system operation. Furthermore, ramping model of the generator outputs and electricity demand could better depict the importance of the consideration of the transient study of the natural gas network. To increase the accuracy of the model, it is necessary to present the power system in dynamic mode or the time step of the UC should break down to less than 1 hour.

\section{REFERENCES}

[1] US energy information administration, "International energy outlook 2016," 2016, accessed 4 July 2017. [Online]. Available: https://www.eia.gov/outlooks/ieo/electricity.php

[2] Y. Ou, H. Zhai, and E. S. Rubin, "Life cycle water use of coal- and natural-gas-fired power plants with and without carbon capture and storage", International Journal of Greenhouse Gas Control, Vol. 44, pp. 249-261, 2016.

[3] B. Zhao, A. J. Conejo, and R. Sioshansi, "Unit Commitment Under GasSupply Uncertainty and Gas-Price Variability", IEEE Transactions on Power Systems, Vol. 32, pp. 2394-2405, Aug. 2016.

[4] C. M. Correa-Posada, and P. Sanchez-Martin, "Security-Constrained Optimal Power and Natural-Gas Flow", IEEE Transactions on Power Systems, Vol. 29, pp. 1780-1787, 2014.

[5] X. Zhang, L. Che, M. Shahidehpour, A. S. Alabdulwahab, and A. Abusorrah, "Reliability-Based Optimal Planning of Electricity and Natural Gas Interconnections for Multiple Energy Hubs", IEEE Transactions on Smart Grid, Vol. 8, pp. 1658-1667, 2017.

[6] Y. Li, Y. Zou, Y. Tan, Y. Cao, X. Liu, M. Shahidehpour, S. Tian, and F. $\mathrm{Bu}$, "Optimal Stochastic Operation of Integrated Low-Carbon Electric Power, Natural Gas, and Heat Delivery System", IEEE Transactions on Sustainable Energy, Vol. 9, pp. 273-283, 2018.

[7] C. Wang, W. Wei, J. Wang, F. Liu, and S. Mei, "Strategic Offering and Equilibrium in Coupled Gas and Electricity Markets", IEEE Transactions on Power Systems, Vol. 33, pp. 290-306, 2017.

[8] A. Alabdulwahab, A. Abusorrah, X. Zhang, and M. Shahidehpour, "Stochastic Security-Constrained Scheduling of Coordinated Electricity and Natural Gas Infrastructures", IEEE Systems Journal, Vol. 11, pp. 1674-1683, 2017.

[9] S. Badakhshan, M. Kazemi, and M. Ehsan, "Security constrained unit commitment with flexibility in natural gas transmission delivery", Journal of Natural Gas Science and Engineering, Vol. 27, pp. 632-640, 2015.

[10] C. Liu, M. Shahidehpour, Y. Fu, and Z.Li, "Security-constrainted unit commitment with natural gas transmission constraints", IEEE Transactions on power systems, VOL, 24, NO. 3, august 2009.

[11] A. Alabdulwahab, A. Abusorrah, X. Zhang, and M. Shahidehpour, "Coordination of Interdependent Natural Gas and Electricity Infrastructures for Firming the Variability of Wind Energy in Stochastic Day-Ahead Scheduling", IEEE Transactions on Sustainable Energy, Vol. 6, pp. 606-615, 2015.

[12] J. Fang, Q. Zeng, X. Ai, Z. Chen, and J. Wen, "Dynamic Optimal Energy Flow in the Integrated Natural Gas and Electrical Power Systems", IEEE Transactions on Sustainable Energy, Vol. 9, pp. 188$198,2018$.

[13] X. Xu, H. Jia, H-D. Chiang, D. C. Yu, and D. Wang, "Dynamic Modeling and Interaction of Hybrid Natural Gas and Electricity Supply System in Microgrid", IEEE Transactions on Power Systems, Vol. 30, pp. 1212-1221, 2015.

[14] N. Noorbehesht, and P. Ghaseminejad, "Numerical simulation of the transient flow in natural gas transmission lines using a computational fluid dynamic method", American journal of applied sciences, 10 (1):2434,2013

[15] B. Zhao, A. J. Conejo, R. Sioshansi, "Coordinated Expansion Planning of Natural Gas and Electric Power Systems", IEEE Transactions on Power Systems, Vol. 33, pp. 3064-3075, 2018.
[16] C. M. Correa-Posada, and P. Sanchez-Martin, "Integrated Power and Natural Gas Model for Energy Adequacy in Short-Term Operation", IEEE Transactions on Power Systems, Vol. 30, pp. 3347-3355, 2015.

[17] C. M. Correa-Posada, and P. Sanchez-Martin, "Security-constrained unit commitment with dynamic gas constraints", Power \& Energy Society General Meeting, 2015 IEEE, DOI: 10.1109/PESGM.2015.7285943.

[18] J. Yang, N. Zhang, C. Kang, and P. Pinson, "Modeling the transient security constraints of natural gas network in day-ahead power system scheduling", IEEE Power \& Energy Society General Meeting, DOI: 10.1109/PESGM.2017.8274497, 2017.

[19] C. M. Correa-Posada and P. Sanchez-Martin, "Gas network optimization: A comparison of piecewise linear models," 2014. [Online]. Available: online.org/DB_HTML/2014/10/4580.html.

[20] P. Duenas, T. Leung, M. Gil, J. Reneses, "Gas-Electricity Coordination in Competitive Markets Under Renewable Energy Uncertainty," IEEE Transactions on Power Systems, Vol. 30, pp. 123-131, 2015.

[21] C. Liu, M. Shahidehpour, and J. Wang, "Coordinated scheduling of electricity and natural gas infrastructures with a transient model for natural gas flow," Chaos: An Interdisciplinary Journal of Nonlinear Science, vol. 21, no. 2, pp. 025 102-025 102, 2011

[22] S. Mokhatab and W. A. Poe, Handbook of natural gas transmission and processing. Gulf Professional Publishing, 2012.

[23] C. Fletcher, Computational Techniques for Fluid Dynamics 2: Specific Techniques for Different Flow Categories. Springer Berlin Heidelberg, 2012.

[24] Y. Zhou, C. Gu, H. Wu, and Y. Song, "An Equivalent Model of Gas Networks for Dynamic Analysis of Gas-Electricity Systems," IEEE Trans. on Power System, Vol. 32, pp. 4255-4264, Nov. 2017.

[25] M. Chaczykowski, "Transient flow in natural gas pipeline c the effect of pipeline thermal model," Applied Mathematical Modelling, vol. 34, no. 4, pp. 1051-1067, 2010.

[26] J. Yang, N. Zhang, C. Kang, and Q. Xia, "Effect of Natural Gas Flow Dynamics in Robust Generation Scheduling Under Wind Uncertainty," IEEE Trans. on Power System, Vol. 33, pp. 2087-2097, March 2018.

[27] S. L. Ke, and H. C. Ti, "Transient analysis of isothermal gas flow in pipeline network", Chemical Engineering Journal, Vol. 76, pp. 169-177, 2000.

[28] M. Behbahani-Nejad, and A. Bagheri, "The accuracy and efficiency of a matlab-simulink library for transient flow simulation of gas pipelines and networks", Journal of Petroleum Science and Engineering, Vol. 70, No. 3, pp. 256-265, 2010.

[29] W. Q. Tao, and H. C. Ti, "Transient analysis of gas pipeline network", Chemical Engineering Journal, Vol. 69, pp. 47-52, 1998.

[30] M. Taherinejad, S. M. Hosseinalipour, and R. Madolit, "Dynamic simulation of gas pipeline networks with electrical analogy", Journal of the Brazilian Society of Mechanical Sciences and Engineering, Vol. 39, pp. 4431-4441, 2017.

[31] M. Mohitpour, H. Golshan, and A. Murray, "Pipeline design and construction," a practical approach, 2nd edn. ASME Press, New York, 2003.

[32] PM. Coelho, and C. Pinho, "Considerations about equations for steady state flow in natural gas pipelines. Journal of the Brazilian Society of Mechanical Sciences and Engineering, Vol. 29, pp. 262-273, 2007.

[33] K. Lin, and K. E. Holbert, "Applying the equivalent pi circuit to the modeling of hydraulic pressurized lines", Mathematics and Computers in Simulation, Vol. 79, pp. 2064-2075, 2009.

[34] N. Chiang, and V. M. Zavala, "Large-scale optimal control of interconnected natural gas and electrical transmission systems", Applied Energy, Vol. 168, pp. 226-235, 2016.

[35] K. Pambour, R. Bolado-Lavin and G. P. J. Dijkema, "An integrated transient model for simulating the operation of natural gas transport systems", Journal of Natural Gas Science and Engineering, Vol. 28, pp. 672-690, 2016.

[36] J. Lin, and F. H. Magnago, Design, Structure, and Operation of an Electricity Market. New York, NY, USA: Wiley-IEEE Press, 2017.

[37] H. Ye and Z. Li, "Robust Security-Constrained Unit Commitment and Dispatch With Recourse Cost Requirement," IEEE Trans. Power Syst, vol. 31, no. 5, pp. 3527-3536, 2016.

[38] H. Üster and S. Dilaveroglu, "Optimization for design and operation of natural gas transmission networks," Applied Energy, vol. 133, pp. 56-69, 2014.

[39] Y. Xu, Y. Ma, X. Zhang, S. Li, Y. Wang, and N. Zhang, "The optimization of gas pipeline transmission and end segment storage in gas network", (ISORA 2013), 11th International Symposium on, DOI: 10.1049/cp.2013.2269.

[40] Available:https://www.motor.ece.iit.edu/data/Gastranssmion_118_14test .xls. 


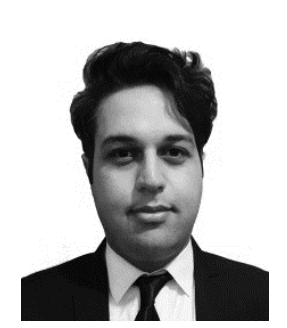

Sobhan Badakhshan received the B.Sc. degree in electrical engineering from Iran University of Science and Technology (IUST), Tehran, Iran, in 2013 and the M. Sc. degree from Sharif University of Technology, Tehran, Iran, in 2015. Currently, he is an associate researcher at the Center of Excellence in Management and Control of Power System, Sharif University of Technology, Tehran, Iran. He is member of the Iran National Elites Foundation. His research interests include economic and secure optimization of electric power systems, energy system optimization, renewable energy integration, smart grids, future energy markets, generation scheduling, operation of integrated energy system, demand side management, and low-carbon power planning.

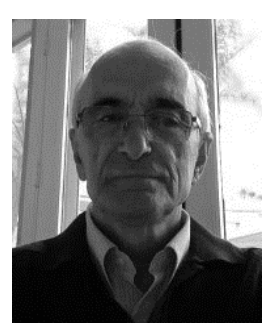

Mehdi Ehsan received the B.Sc. and M.Sc. degrees in electrical engineering from Technical College of Tehran University, Tehran, Iran, in 1963, and the $\mathrm{Ph} . \mathrm{D}$. and DIC degrees in power engineering from Imperial College University London, London, U.K., in 1976. Currently, he is a Professor in the Electrical Engineering (EE) Department of Sharif University of Technology (SUT), Tehran and dean of the Center of Excellence in Management and Control of the Power System in the EE Department of SUT, Tehran. He has extended cooperation with research centers in

Iran and also abroad.

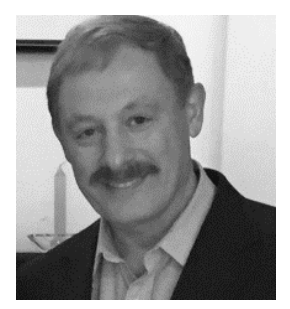

Mohammad Shahidehpour (F'01) received an Honorary DSc degree from the Polytechnic University of Bucharest, Romania. $\mathrm{He}$ is a University Distinguished Professor and Bodine Chair Professor at Illinois Institute of Technology where he also serves as Director of the Robert W. Galvin Center for Electricity Innovation. He is the recipient of the 2019 IEEE PES Ramakumar Family Renewable Energy Excellence Award. Dr. Shahidehpour is a Fellow of the American Association for the Advancement of Science (AAAS), Fellow of IEEE, and Fellow of the National Academy of Inventors (NAI). He is member of the US National Academy of Engineering.

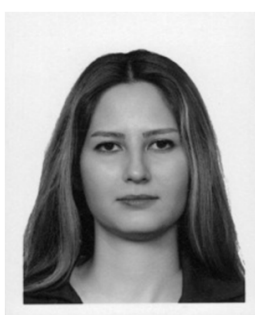

Neda Hajibandeh (S'17) received the M.Sc. degree in electrical engineering from Tarbiat Modares University, Tehran, Iran, in 2011. She is a PhD candidate at University of Beira Interior (UBI), Covilha, Portugal since 2016 while working on the project named 'Enhancing Smart GRIDS for Sustainability' at CMAST/UBI. Currently, as a $\mathrm{Ph} . \mathrm{D}$. student in electrical engineering - power systems - with professional experience in substation protection and control design, power system planning and power system restructuring, she combines organized electricity markets behaviors with an emphasis on renewable energies, demand response programs and social sustainability.

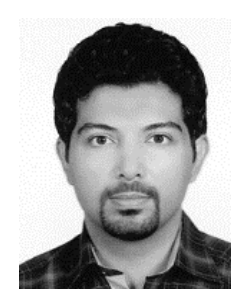

Miadreza Shafie-khah (M'13-SM'17) received the M.Sc. and Ph.D. degrees in electrical engineering from Tarbiat Modares University, Tehran, Iran, in 2008 and 2012, respectively. He received his first postdoc from the University of Beira Interior (UBI), Covilha, Portugal in 2015. He received his second postdoc from the University of Salerno, Salerno, Italy in 2016. Currently, he is an Assistant Professor at the University of Vaasa, Vaasa, Finland. He was considered one of the Outstanding Reviewers of the IEEE TRANSACTIONS ON SUSTAINABLE ENERGY, in 2014 and 2017, one of the Best Reviewers of the IEEE TRANSACTIONS ON SMART GRID, in 2016 and 2017, and one of the Outstanding Reviewers of the IEEE TRANSACTIONS ON POWER SYSTEMS, in 2017 and 2018. His research interests include power market simulation, market power monitoring, power system optimization, demand response, electric vehicles, price forecasting and smart grids.

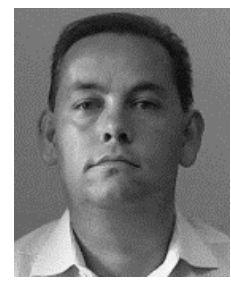

João P. S. Catalão (M'04-SM'12) received the M.Sc. degree from the Instituto Superior Técnico (IST), Lisbon, Portugal, in 2003, and the Ph.D. degree and Habilitation for Full Professor ("Agregação") from the University of Beira Interior (UBI), Covilha, Portugal, in 2007 and 2013, respectively.

Currently, he is a Professor at the Faculty of Engineering of the University of Porto (FEUP), Porto, Portugal, and Research Coordinator at INESC TEC. He was also appointed as Visiting Professor by North China Electric Power University, Beijing, China. He was the Primary Coordinator of the EU-funded FP7 project SiNGULAR ("Smart and Sustainable Insular Electricity Grids Under Large-Scale Renewable Integration"), a 5.2-million-euro project involving 11 industry partners. He has authored or coauthored more than 700 publications, including 275 journal papers (more than 80 IEEE Transactions/Journal papers), 372 conference proceedings papers, 5 books, 35 book chapters, and 14 technical reports, with an $h$-index of 47 , an $i 10$-index of 197, and over 8500 citations (according to Google Scholar), having supervised more than 70 post-docs, Ph.D. and M.Sc. students. He is the Editor of the books entitled "Electric Power Systems: Advanced Forecasting Techniques and Optimal Generation Scheduling" and "Smart and Sustainable Power Systems: Operations, Planning and Economics of Insular Electricity Grids" (Boca Raton, FL, USA: CRC Press, 2012 and 2015, respectively). His research interests include power system operations and planning, hydro and thermal scheduling, wind and price forecasting, distributed renewable generation, demand response and smart grids.

Prof. Catalão is an Editor of the IEEE TRANSACTIONS ON SMART GRID, an Editor of the IEEE TRANSACTIONS ON POWER SYSTEMS, an Associate Editor of the IEEE TRANSACTIONS ON INDUSTRIAL INFORMATICS. From 2011 till 2018 (seven years) he was an Editor of the IEEE TRANSACTIONS ON SustaINABLE ENERGY and an Associate Editor of the IET Renewable Power Generation. He was also a Subject Editor of the IET Renewable Power Generation. He was the Guest Editor-in-Chief for the Special Section on "Real-Time Demand Response" of the IEEE TRANSACTIONS ON SMART GRID, published in December 2012, the Guest Editor-in-Chief for the Special Section on "Reserve and Flexibility for Handling Variability and Uncertainty of Renewable Generation" of the IEEE TRANSACTIONS ON SUSTAINABLE ENERGY, published in April 2016, the Corresponding Guest Editor for the Special Section on "Industrial and Commercial Demand Response" of the IEEE TRANSACTIONS ON INDUSTRIAL INFORMATICS, published in November 2018, and the Lead Guest Editor for the Special Issue on "Demand Side Management and Market Design for Renewable Energy Support and Integration" of the IET Renewable Power Generation, published in April 2019. He was the recipient of the 2011 Scientific Merit Award UBIFE/Santander Universities, the 2012 Scientific Award UTL/Santander Totta, the 2016 and 2017 FEUP Diplomas of Scientific Recognition, the 2017 Best INESC-ID Researcher Award, and the 2018 Scientific Award ULisboa/Santander Universities, in addition to an Honorable Mention in the 2017 Scientific Award ULisboa/Santander Universities. Moreover, he has won 4 Best Paper Awards at IEEE Conferences. 